\title{
La carte génétique de l'homme enfin sur orbite... de microsatellites
}

En dépit du nombre impressionnant de cartes génétiques de chromosomes humains proposées ces dernières années, la carte génétique de l'homme souffrait encore récemment de plusieurs défauts majeurs : (1) les cartes de liaison étaient surtout fondées sur la localisation de RFLP (restriction fragment length polymorphisms) qui s'avèrent d'unc utilité limitée dans la cartographie des maladies génétiques. Ces RFLP, pour la plupart bi-alléliques, ont permis de cartographier les affections génétiques les plus fréquentes, mais sont insuffisants dans le cas de maladies plus rares ou hétérogènes sur le plan génétique ; (2) certaincs régions chromosomiques demcurent encore mal couvertes et il faut souvent plusieurs années pour définir un intervalle génétique minimal fondé sur la définition des points de recombinaison à partir d'une localisation primaire; (3) la mise en évidence des loci à effet mineur dans les maladies multifactorielles, repose sur une carte génétique à hautc densité. Il y a donc à la fois nécessité de développer un grand nombre de marqueurs génétiques et de disposer de marqueurs hautement informatifs.

En raison de la faible informativité des marqueurs bi-alléliques on s'oriente de plus en plus systématiquement vers la recherche de marqueurs multialléliques. De courtes répétitions en tandem (10 à 30 fois) de séquences nucléotidiques très simples (di-, tri-, tétranucléotides, etc.) sont disséminées en plusieurs dizaines de milliers d'emplacements distincts du génome des mammifères. Ces motifs, encore appelés microsatellites ou STR (simple ou short tandem repeats), sont fréquemment multi-alléliques: les variations correspondent à des changements du nombre des répétitions à l'intérieur d'un motif $\left(\mathrm{m} / \mathrm{s} n^{\circ} 6\right.$, vol. $6, p$. 576). Ces changements de taille de quelques paires de bases sont mis en évidence après amplification par PCR du locus sur gel d'acrylamide dénaturant. Cette méthode de détection de loci polymorphes [1, 2] est cependant lourde : elle nécessite l'acquisition de données de séquence des régions flanquant les motifs polymorphes et requiert l'utilisation de systèmes analytiques contraignants (marquages radioactifs, gels d'acrylamide dénaturants). Malgré ces inconvénients, l'utilisation généralisée de ce type de marqueurs en cartographie génétique est en train de modifier radicalement l'état des cartes génétiques chez les mammifères.

Ainsi chez l'homme, deux cartes de liaison génétique de l'ensemble du génome viennent d'être publiées à moins d'un mois d'intervalle. Unc première série de travaux patronnée par le NIH ct le CEPH a permis d'intégrer dans des cartes surtout constituées d'anciens RFLP bi-alléliques, 340 marqueurs microsatellites dont la moitié dépasse le seuil d'hétérozygotie de $70 \%$ [3]. Une nouvelle cartc génétique de deuxième génération entièrement constitutée de marqueurs microsatellites dont les trois quarts ont une hétérozygotic supérieure à $70 \%$ vient d'être proposée par le laboratoire Généthon [4]. Les distances génćtiques couvertes par cette dernière sont notablement plus faibles quc celles publiées par le NIH/CEPH, mais plus proches des valeurs attendues théoriquement. Ces différences sont principalement dues aux erreurs de génotypage assez courantes et difficilement contrôlables lorsque les cartes sont établics à partir de données disparates comme c'est le cas de la carte NIH/CEPH. Les erreurs ont pour effet d'augmenter artificiellement le nombre de recombinaisons et donc les distances génétiques.

La très grande majorité des cartes génétiques est établie à partir des génotypes des $\mathrm{ADN}$ de familles de référence rassemblées par le CEPH ( $\mathrm{m} / \mathrm{s} n^{\circ} 3$, vol. 6, p. 286). L'utilisation de ces ADN de référence permet d'étudier les mêmes méioses dans des laboratoires différents (une centaine actuellement) et de préciser les quelques 50 à 60 événements de recombinaison d'un génome diploïde à partir des résultats provenant de l'ensemble de la communauté des cartographes et rassemblés dans une base de données commune maintenue par le CEPH, les deux cartes mentionnées ici ont été établies à partir des génotypes des familles du CEPH.

Le niveau de résolution de ces cartes dépend du nombre de méioses informatives analysécs. En raison de l'important déchet de génotypages non informatifs des marqueurs bi-alléliques, les collaborateurs du CEPH s'étaient cngagés à analyser un total de 40 familles. Lorsque le taux d'hétérozygotes observés augmente, on peut obtenir une résolution de même niveau en établissant le génotype de moins de familles. Le génotypage de huit familles du CEPH a permis à l'équipe de Généthon d'ordonner 550 marqueurs avec un rapport de vraisemblance supérieur à 1000 , dépassant souvent celui obtenu avec 40 familles pour des marqueurs bi-alléliques. En outre, l'analyse d'un nombre plus important de familles ne permet pas des gains de résolution importants, notamment pour l'ordonnancement de marqueurs séparés par de très faibles distances dc recombinaison. Il scra sans doute plus aisé d'ordonner ces marqueurs sur unc carte physique $\left(\mathrm{m} / \mathrm{s} n^{\circ} 8\right.$, vol. $8, p$. 881).

Le projet de cartographie génétique de Généthon repose sur quelques options destinées à mener de manière intensive un ensemble de manipulations propres aux laboratoires de recherche : clonage, criblage de banques, séquençage, analyse informatique de séquences, amplification par PCR, génotypage, analyse ct traitement des données de génotypage. Parmi les nombreux motifs microsatellites existants, lc motif CA est de loin le plus fréquent. C'est aussi celui dont les caractéristiques sont 
les mieux connues. Il n'existe pas à cc jour d'étude systématique permettant de connaître avec précision le degré de polymorphisme des autres motifs microsatcllites. Pour ces diverses raisons, seul le motif CA a ćté retenu pour l'établissement de cettc carte.

Afin de réduire le nombre de gels de séquence, une technique en "multiplex " dérivéc de la méthode de séquençage de Church et KiefferHiggins [5] a été utiliséc pour le déterminisme du génotype. Les produits de PCR de huit à 16 marqueurs différents d'un même individu ont été mélangés, mis à co-migrer sur unc sculc piste de gel, puis transférés sur membranc. Les produits d'amplification ont ensuitc été détectés par hybridation à des sondes non radioactives correspondant à unc des amorces allongée puis couplée à la peroxydase. Cependant, pour pouvoir démarrer ce projet le plus rapidement possible, aucune automatisation ad hoc n'a ćté développée ct les manipulations sont pour l'essentiel menées manucllement avec l'aide d'automates du commerce. En raison du volume des donnćes à traiter il a nćanmoins ćté indispensable d'automatiser l'enchaînement des opérations de calcul de linkage et de construction des cartes.

L'établissement de cette carte génétique n'est que dans sa première phase. Une carte au nivcau de résolution obtenu cst déjà d'une utilité importante dans la cartographic primaire de maladics génétiques à transmission mendélicnnc, puisqu'elle met à la disposition des généticiens, pour des études de linkage ; un ensemble de marqucurs régulièrement espacés et permettant de couvrir la quasi-totalité du génome. Il s'est donc avéré indispensable de publier cette première carte partielle. Cellc-ci, d'unc résolution moyenne de $6 \mathrm{cM}$ présente encore une douzaine de régions de plus de $20 \mathrm{cM}$ dépourvues de microsatellites. Certains des intervalles non couverts ont aussi ćté observés sur les autres cartes. Il n'est donc pas exclu que ces régions soicnt associćes à une " recombinogénicité " particulièrement élevéc ayant pour effet d'amplifier les distances génćtiques par rapport aux distances physiques. Cela a déjà été observé pour des régions subtćlomériques et, de fait, certaines font partie des intervalles mal couverts.

$\mathrm{m} / \mathrm{s} n^{\circ} 1$ vol. 9, janvier 93
L'établissement d'une carte à 2000 marqueurs est en cours. La plupart des grands intervalles ont déjà été réduits par l'analyse génotypique de nouveaux marqueurs obtenus au hasard. Mais il est possible que l'obtention d'une carte homogène à haute densité nécessite de faire appel à des approches ciblées ou à l'application de stratégies particulières permettant l'isolement préférentiel de marqueurs de régions mal couverts. Certaines de ces stratégies sont en cours d'évaluation.

A côté d'une carte génćtique plus dense, il devient impératif d'établir un lien entre cette carte génćtique ct les segments contigus de clones de YAC chevauchants obtenus par la méthode d'analyse des fingerprints récemment décrite [6] $\left(\mathrm{m} / \mathrm{s} n^{\circ} 8\right.$, vol. 8, p. 881). Cela constituera la première version d'une carte intégrée qui devrait avoir un impact considérable dans la cartographie des maladies génétiques et servir de point de départ à une collection de segments ordonnés et chevauchants d'ADN cloné représentant l'ensemble du génome humain

\section{RÉFÉRENCES}

1. Weber JL, May PE. Abundant class of human DNA polymorphisms which can bc typed using the polymerase chain reaction. $\Lambda \mathrm{m}$ J Hum Genet 1989 ; 44 : 388-96.

2. Litt M, Luty JA. A hypervariable microsatellite revealed by in vitro amplification of a dinuclcotide repeat within the cardiac muscle actin gene. Am J Hum Gonet 1989; 11: $397-401$.

3. NIH/CEPH Collaborative Mapping Group. A comprehensive genetic linkage map of the human genomc. Science $1992 ; 258$ : 67-86.

4. Weissenbach J, Gyapay G, Dib G, et al. A sccond-gencration linkage map of the human genomc. Nature 1992 ; 359 : 794-801.

5. Church GM, Kieffer-Higgins S. Multiplex DNA scquencing. Science $1988 ; 240: 185-8$. 6. Bellannć-Chantelot C, Lacroix B, Ougen $\mathrm{P}$, et al. Mapping the wholc genome by fingerprinting yeast artificial chromosomes. Cell $1992 ; 70$ : 1059-68.

Jean Weissenbach

Cnrs URA 1445, Institut Posteur, 25, nue du Docteur Roux, 75724 Paris Cedex 15, France.

TIRÉS A PART

J. Wcissenbach. 